\title{
Intra-aortic balloon pump inserted through the subclavian artery: A minimally invasive approach to mechanical support in the ambulatory end-stage heart failure patient
}

\author{
Mark J. Russo, MS, MD, ${ }^{\text {a,b }}$ Valluvan Jeevanandam, MD, ${ }^{\mathrm{b}}$ John Stepney, PAC, ${ }^{\mathrm{a}, \mathrm{b}}$ Aurelie Merlo, ${ }^{\mathrm{a}}$ \\ Elizabeth M. Johnson, MA, ${ }^{\mathrm{a}, \mathrm{b}}$ Raja Malyala, MD, ${ }^{\mathrm{c}}$ and Jai Raman, MD, FRACS, $\mathrm{PhD}^{\mathrm{c}}$
}

\begin{abstract}
Objective: Intra-aortic balloon pumps are traditionally inserted through the femoral artery, limiting the patient's mobility. We used alternate approaches of intra-aortic balloon pump insertion to provide temporary and minimally invasive support for patients with decompensating, end-stage heart failure. The present study describes the outcomes with closed-chest, transthoracic intra-aortic balloon pumps by way of the subclavian artery.
\end{abstract}

\begin{abstract}
Methods: During a 3-year period, 20 patients underwent subclavian artery-intra-aortic balloon pump in the setting of end-stage heart failure. The balloon was inserted through a polytetrafluoroethylene graft sutured to the right subclavian artery in 19 patients $(95 \%)$ and to the left subclavian artery in 1 patient $(5 \%)$. The goal of support was to bridge to transplantation in 17 patients $(85 \%)$ and bridge to recovery in 3 patients $(15 \%)$. The primary outcome measure was death during subclavian artery-intra-aortic balloon pump support. The secondary outcomes included survival to the intended endpoint of bridge to transplantation/bridge to recovery, complications during subclavian artery-intra-aortic balloon pump support (eg, stroke, limb ischemia, brachial plexus injury, dissection, bleeding requiring reoperation, and device-related infection), emergent surgery for worsening heart failure, and ambulation during intra-aortic balloon pump support.
\end{abstract}

Results: The duration of balloon support ranged from 3 to 48 days (mean, $17.3 \pm 13.1$ days). No patients died during subclavian artery-intra-aortic balloon pump support. Of the 20 patients, $14(70 \%)$ were successfully bridged to transplant or left ventricular-assist device. Two patients $(10 \%)$ required emergent left ventricularassist device for worsening heart failure.

Conclusions: An intra-aortic balloon pump inserted through the subclavian artery is a simple, minimally invasive approach to mechanical support and is associated with limited morbidity and facilitates ambulation in patients with end-stage heart failure. (J Thorac Cardiovasc Surg 2012;144:951-5)

The intra-aortic balloon pump (IABP) was first used successfully by Kantrowitz and colleagues ${ }^{1}$ in 1968 for patients with cardiogenic shock. Annually, tens of thousands of patients benefit from the use of IABPs for indications including refractory angina pectoris, post-cardiopulmonary bypass shock, temporizing complications of percutaneous coronary intervention, and complications of myocardial infarction refractory to pharmacologic therapy. ${ }^{2}$

Conventionally, IABPs are placed using femoral artery access. However, this approach is associated with a number of important limitations. Most significantly, it requires bed

\footnotetext{
From the Center for Aortic Diseases, ${ }^{\mathrm{a}}$ and Section of Cardiac and Thoracic Surgery, ${ }^{\mathrm{b}}$ Department of Surgery, University of Chicago, Chicago, Ill; and Division of Cardiac Surgery, ${ }^{c}$ Rush University, Chicago, Ill.

Disclosures: Authors have nothing to disclose with regard to commercial support.

Presented at the 25th Meeting of the European Association for Cardio Thoracic Surgery, Lisbon, Portugal, October 1-5, 2011.

Received for publication Jan 3, 2012; revisions received Feb 21, 2012; accepted for publication March 12, 2012; available ahead of print April 23, 2012.

Address for reprints: Mark J. Russo, MS, MD, Section of Cardiac and Thoracic Surgery, University of Chicago Medical Center, E-500, 5841 South Maryland Avenue, Chicago, IL 60637 (E-mail: mrusso@uchicago.edu).

$0022-5223 / \$ 36.00$

Copyright (c) 2012 by The American Association for Thoracic Surgery doi:10.1016/j.jtcvs.2012.03.007
}

rest-precluding ambulation. Second, there is a significant risk of leg ischemia, with a reported incidence of $5 \%$ to $19 \%{ }^{2}$

Given these limitations, alternative approaches, including by way of the subclavian artery (SCA), have been explored. Although technically more demanding and more time consuming, the SCA approach overcomes important limitations of femoral placement, because the SCA is generally free of atherosclerosis, even in patients with significant peripheral arterial disease. Furthermore, it allows patients to ambulate early. SCA placement of IABPs was described by Mayer $^{3}$ in 1978. McBride and colleagues ${ }^{4}$ described ambulatory use of the balloon pump, and Cochran and colleagues ${ }^{5}$ reported their ambulatory technique in 4 patients awaiting transplantation.

In the present series, we report a 3-year experience with SCA-IABP at a single institution. This is the largest experience with SCA-IABPs published to date. The approach we describe includes important technical modifications we have developed during our growing experience.

\section{METHODS \\ Data Collection}

The present study was in accordance with the University of Chicago's institutional review board. The patient data, including preprocedure 


\section{Abbreviations and Acronyms \\ $\mathrm{CABG}=$ coronary artery bypass grafting \\ IABP $=$ intra-aortic balloon pump \\ LVAD $=$ left ventricular assist device \\ SCA = subclavian artery}

characteristics and outcomes, were obtained from our institutional cardiac surgery outcomes database. Among the 20 patients receiving SCA-IABP support, $15(75 \%)$ were men and $5(25 \%)$ were women. Their age ranged from 20 to 70 years (mean, $57.8 \pm 13.0$ years).

\section{Study Population}

The analysis included 20 consecutive patients who underwent SCAIABP principally to support heart failure while awaiting cardiac transplantation. Other indications included deteriorating hemodynamics in patients who were not transplant or ventricular assist device candidates and in 1 instance with concomitant coronary artery bypass grafting (CABG) when it was anticipated that the balloon might be necessary for an extended period.

\section{Statistical Analysis}

The patients were followed up from the date of SCA-IABP insertion to the date of device removal, transplant, insertion of a ventricular assist device, or death. The date of the last known follow-up examination was September 28, 2011.

\section{Operative Technique}

In each instance, either the right (17 patients) or, less frequently, the left SCA (3 patients)—when access through the right was not possible-was isolated through a small incision in the infraclavicular region. After $5,000 \mathrm{U}$ of heparin, a side-biting clamp was applied to the SCA. A 4 by 7-mm, tapered polytetrafluoroethylene graft (Gore-Tex, W. L. Gore \& Associates, Inc, Flagstaff, Ariz) was used. An incision was made $8 \mathrm{~cm}$ from the most narrow portion and another $1.5 \mathrm{~cm}$ from the proximal end. This was to ensure that the 1-way stopper slid over the distal portion and was at the level of the skin without stretching or kinking. This was then anastomosed to the artery using 6-0 polypropolene suture on a small needle. As previously described, ${ }^{6}$ and as shown in Figure 1, the 1-way valve was obtained from the $8 \mathrm{~F}$ introducer sheath in our standard Maquet IABP catheter kit (the sheath was cut at the hub, the side hole was cut off, the inner metal coil was removed to prevent balloon rupture), and the 1-way valve was placed on the graft and secured with three 2-0 silk ties. Under fluoroscopic guidance, the glide wire was then inserted through the needle into the graft and then through the SCA into the aorta.

Occasionally, the wire will pass directly into the descending aorta. On other occasions, the wire will head preferentially into the ascending aorta. The following procedure can be useful in redirecting the catheter:

1. Place a right coronary artery Amplatz catheter (AGA Medical, Plymouth, Minn) in the ascending aorta and work the catheter leftward and upward, toward the arch.

2. Use an Omniflush (AngioDynamics, Latham, NY) catheter (reverse angle catheter) and non-stiff glide wire. Once the wire is pointed in the appropriate direction, switch out the Omniflush for a glide catheter or quick-cross catheter; and direct the wire into descending aorta.

3. If a type 1 or 2 arch is present and access is from the right, using a directional hydrophilic (eg, angled glide wire) and simple curved catheter (eg, Kumpe [Cook, Bloomington, Ind] or Bernstein [AngioDynamics]), advance the catheter into the arch and direct the catheter down the arch.

4. If a type 3 arch is present and access is from the right, a complex curved catheter (eg, Simmons 1 [Cook]) can be advanced down the ascending aorta and then pulled back to engage the tip on the greater curve.
5. As a last resort, the catheter can be placed in the left ventricle; then, using the inferior wall to support the catheter, a stiff wire can be inserted upward back through the aortic valve into the ascending aorta. The wire then typically goes into the arch and then into the descending aorta.

Next, the balloon is placed over the wire to a point $2 \mathrm{~cm}$ below the left SCA. The distal end should be placed just distal to the left subclavian. This can typically be confirmed by both transesophageal endocardiography and fluoroscopy. The wound over the polytetrafluoroethylene graft (Gore-Tex, W. L. Gore \& Associates) is closed in layers. Therefore, the only foreign material that traverses the skin is the balloon catheter, which does so through a separate puncture site below the incision.

\section{Outcomes}

The primary outcome of interest was death during SCA-IABP support. The secondary endpoints included survival to transplantation or recovery without the need for additional mechanical support (eg, ventricular assist device), interval to ambulation, stroke, bleeding requiring reoperation, limb ischemia, other vascular complications, and device failure.

\section{RESULTS \\ Study Population and Indications}

The intent of the IABP was as a bridge to transplantation in 17 patients, and 3 (patients 3, 9, and 17) had a non-bridge to transplantation indication. The intent was to either achieve recovery or temporize pending decisions about more definitive therapy. One of these patients (patient 3) was a 66-year-old patient who had undergone CABG and repair of a postinfarct ventricular septal defect, who was readmitted with a leak from the ventricular septal defect patch and sepsis. An IABP in the subclavian position was a bridge to recovery because he was not a candidate for either a ventricular assist device or transplantation because of the sepsis. A second patient (patient 9) was a 65-year-old man who presented with cardiogenic shock after acute anterior wall infarction. After percutaneous transluminal coronary angioplasty, a femoral IABP and a tandem heart were placed for support. A SCA-IABP was later placed for long-term support in exchange for the femorally placed devices. Because of a history of Alzheimer's disease, he was not a candidate for ventricular assist device or transplantation. The third patient (patient 17) was a 57-year-old man with triple vessel disease and an ejection fraction of $15 \%$. He underwent subclavian IABP with concomitant CABG.

\section{Patient Management}

Of the 20 patients, $9(45 \%)$ underwent the procedure under local anesthesia and $11(55 \%)$ required general anesthesia. Of the 20 patients, $6(30 \%)$ received no anticoagulation or only prophylactic subcutaneous heparin doses postoperatively, and $14(70 \%)$ received anticoagulation with intravenous heparin, low-molecular-weight heparin, or warfarin.

\section{Primary Outcomes}

As summarized in Table 1, the duration of balloon support ranged from 3 to 48 days (mean, $17.3 \pm 13.1$ days). No patients died during SCA-IABP support. 


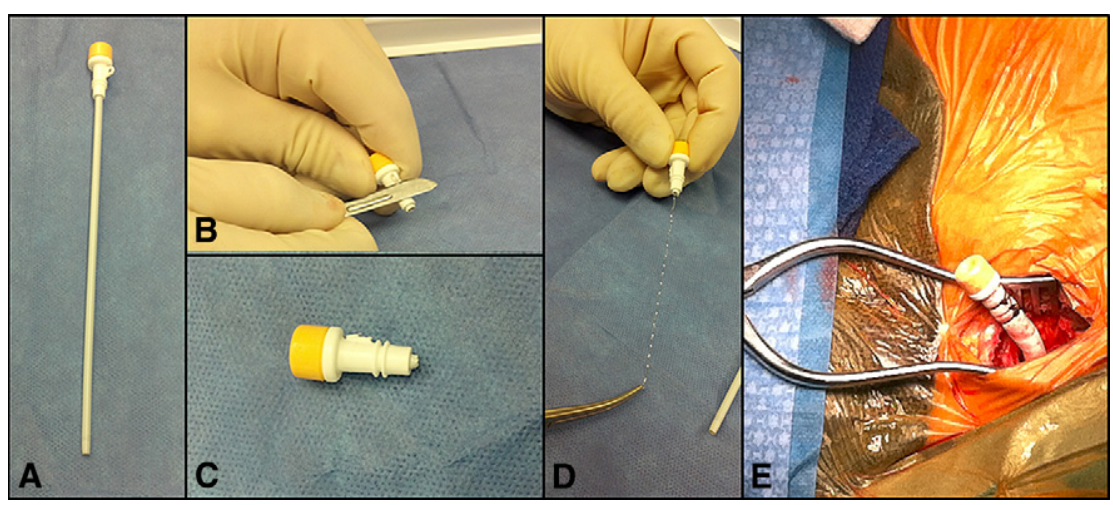

FIGURE 1. A-E, Method used to obtain and secure the 1-way valve to the graft.

\section{Secondary Outcomes}

Of the 17 patients who had undergone the procedure as a bridge to transplantation, $14(82.3 \%)$ eventually underwent transplantation. Two patients $(11.7 \%)$ required a ventricular assist device for bridging. The first patient (patient 15) required an assist device 18 days after implantation because of worsening heart failure. The second patient (patient 8) required biventricular support 26 days after balloon placement. In each case, the balloon was removed after placement of these devices. A third patient (patient 19) was awaiting transplantation 5 weeks after IABP implantation.

As summarized in Table 2, 3 cases of device failure occurred. In 1 case, the middle of the balloon was not filling through the proximal and distal ends. In 1 case, the balloon

TABLE 1. Case studies of 20 patients who underwent SCA-IABP insertion during a 3-year period

\begin{tabular}{lllllc}
\hline $\begin{array}{l}\text { Pt. } \\
\text { no. }\end{array}$ & Goal & $\begin{array}{c}\text { Age } \\
(\mathbf{y})\end{array}$ & Outcome & $\begin{array}{c}\text { Interval from IABP } \\
\text { to outcome (d) }\end{array}$ & $\begin{array}{c}\text { Duration of } \\
\text { IABP support (d) }\end{array}$ \\
\hline 1 & BTT & 62 & TX & 2 & 6 \\
2 & BTT & 63 & TX & 1 & 7 \\
3 & Recovery & 66 & IABP out & 24 & 24 \\
4 & BTT & 58 & TX & 12 & 14 \\
5 & BTT & 67 & TX & 13 & 16 \\
6 & BTT & 56 & TX & 48 & 48 \\
7 & BTT & 70 & TX & 1 & 3 \\
8 & BTT & 20 & BiVAD & 26 & 26 \\
9 & Recovery & 65 & IABP out & 9 & 9 \\
10 & BTT & 65 & TX & 7 & 8 \\
11 & BTT & 45 & TX & 6 & 8 \\
12 & BTT & 61 & TX & 10 & 11 \\
13 & BTT & 62 & TX & 43 & 44 \\
14 & BTT & 29 & TX & 6 & 22 \\
15 & BTT & 66 & LVAD & 18 & 19 \\
16 & BTT & 57 & TX & 12 & 13 \\
17 & Recovery & 57 & IABP out & 6 & 6 \\
18 & BTT & 69 & TX & 2 & 47 \\
19 & BTT & 50 & Ongoing & - & 20 \\
20 & BTT & 67 & TX & 19 & \\
\hline
\end{tabular}

SCA, Subclavian artery; IABP, intra-aortic balloon pump; Pt. no., patient number; $B T T$, bridge to transplantation; $T X$, transplantation; $B i V A D$, biventricular assist device. appeared to develop a leak. Finally, in the third case, although the device appeared to be functioning properly, the expected augmentation was suboptimal. Therefore, the device was exchanged, and augmentation improved.

Three patients (patients 3, 9, and 17) underwent the procedure as a bridge to recovery for other indications. In all $3 \mathrm{pa}-$ tients, the balloon was able to be removed. Patient 17, who had his device placed intraoperatively during high-risk CABG, was successfully discharged. However, 2 patients died several days (10 and 13 days) after removal of the balloon pump; it had been determined that they were not candidates for more long-term assistance or transplantation.

Except in the instances in which the patient was ventilator dependent preoperatively, all patients were extubated within 24 hours after the procedure. Fourteen patients $(70 \%)$ were able to ambulate within 1 to 5 days after insertion (mean, $2.6 \pm 1.2$ days). Three (15\%) of the remaining 6 underwent transplantion within 2 days after IABP insertion; thus, the IABP did not limit ambulation. The remaining 3 patients $(15 \%)$ did not ambulate- this was because they were ventilator dependent or required multiple intravenous lines. No patients developed bleeding requiring reoperation, devicerelated infection, stroke, brachial plexus injury, ischemic limb complications, or other vascular complications.

\section{DISCUSSION}

The support of patients with decompensating end-stage heart failure remains a dilemma. Inotropic therapy is limited by the associated arrhythmias and potential for tolerance. Mechanical support using ventricular assist devices is highly invasive, associated with significant adverse events, and extremely costly. During the past 4 decades, IABPs have compiled a long track record of successfully supporting patients with decompensated heart failure. It has a number of attractive characteristics. The cost is relatively low. It can be inserted by the bedside, in the cardiac catheterization laboratory, in the operating room, or in the ward, if needed. IABPs are available in most countries worldwide where interventional cardiology has achieved some degree of sophistication. 
TABLE 2. Outcomes of 20 patients who underwent SCA-IABP insertion during a 3-year period

\begin{tabular}{lc}
\hline \multicolumn{1}{c}{ Outcome of interest } & Patients (n) \\
\hline Total patients & $20(100)$ \\
Death during IABP support & $0(0)$ \\
Primary endpoint & $17(85)$ \\
Device failure & $3(15)$ \\
Bleeding requiring reoperation & $0(0)$ \\
Device related infection & $0(0)$ \\
Brachial plexus injury & $0(0)$ \\
Stroke & $0(0)$ \\
Limb ischemia & $0(0)$ \\
Other vascular complications & $0(0)$ \\
\hline Data in parentheses are percentages. SCA, Subclavian artery; IABP, intra-aortic bal- \\
loon pump.
\end{tabular}

However, IABPs have limitations, largely related to the use of femoral access. First, IABPs with femoral insertion require that the femoral and iliac arteries are free of significant obstructive disease. In addition, groin catheters prevent ambulation. The patient is not allowed to bend that hip, which also usually prevents the patient from sitting upright. Furthermore, despite the widespread use of sheathless insertion of IABPs, there is a significant risk of ischemia to the limb. Finally, femoral catheters can be associated with greater infection rates ${ }^{7}$ than alternative access sites.

Alternative sites of IABP insertion have occasionally been reported. The SCA approach was first proposed by Mayer $^{3}$ in 1978. He suggested subperiosteal wedge resection of the clavicle to gain access to the SCA. Only 1 clinical report to date has mentioned the use of the $\mathrm{SCA}^{5}$ in any sustained fashion. In that experience, 4 patients with significant aortoiliac disease underwent IABP insertion through the SCA approach jointly by cardiologists and vascular surgeons.

Using the approach developed in the present series, SCAIABP is a relatively simple technique and offers another tool in the mechanical support of patients with cardiogenic shock. For transplant candidates, SCA-IABPs are particularly useful in patients who have previously undergone cardiac surgery, patients listed for multiorgan transplantation, patients requiring repeat transplant, or patients with a contraindication to anticoagulation and are therefore candidates for a ventricular assist device. The procedure can be performed with the patient under local anesthesia. Furthermore, the use of the previously described 1-way valve unique at our institution ${ }^{6}$ allows safe removal or exchange at the bedside without vascular compromise to the arm. Additional advantages of the SCA graft approach include no special restrictions in terms of position in bed and the opportunity to ambulate. This not allows only for more freedom of movement, but also the possibility for the deconditioned patient to participate in physical therapy and rehabilitation.
We have a large and increasing experience with implantable ventricular assist devices. However, the percentage of these patients who had an indication of bridge to transplantation was likely lower than at comparable centers. This might be in part because increasingly we are using SCAIABP as a mechanical circulatory support for bridge to transplantation. For example, from 2009 to 2010, we implanted 93 left ventricular assist devices (LVADs); 13 LVAD patients underwent transplantation; and 11 SCAIABP patients underwent transplantation. Furthermore, we are developing more experience with SCA-IABP as a bridge to recovery in patients with a low ejection fraction with ischemic cardiomyopathy after standard cardiac surgery procedures. This allows $\mathrm{CABG}$ and valve patients to ambulate in the intensive care unit during their recovery after cardiac surgery. Anecdotally, they have lower inotrope and vasoactive medication requirements. Finally, recently, we have successfully used SCA-IABP to optimize preventricular assist device patients before implantation.

In the present series, the largest described series to date, no patients died during SCA-IABP support-although 2 patients required conversion to a LVAD. Furthermore, there were no vascular complications, device-related infections, strokes, or episodes of bleeding requiring reoperation. Importantly, there were also no instances of bleeding from the graft site from this technique. It is noteworthy that most patients underwent transplantion within 2 weeks of IABP insertion. This is in part because patients with IABPs are generally high-priority candidates with a status $1 \mathrm{~A}$ listing. Also, unlike patients undergoing implantable ventricular assist devices, they do not require any significant recovery time after implantation and before they are appropriate for transplantation.

Using the left SCA is generally less desirable because patients with end-stage heart failure often have automated implantable cardioverter defibrillators in the left subclavian vein. In 3 patients, the left subclavian was used because the right was less desirable given the presence of an automated implantable cardioverter defibrillator in 1 case, known left subclavian stenosis in 1 case, and a dialysis catheter in 1 case. On 2 occasions, we have placed SCA-IABPS on the same side as an automated implantable cardioverter defibrillator without complications. Most of this experience has described patients who were candidates for transplantation. However, in older patients with ectactic and atherosclerotic aortas, the left subclavian approach might be preferable. Furthermore, additional imaging of the aorta to assess for calcium might help risk stratify patients who are at high risk of embolization and stroke.

We had 3 cases of device failure. In each case, the device was exchanged over a wire without incident. Nevertheless, the IABP does pose a risk to the patient; therefore, the function of the entire balloon should be confirmed under fluoroscopy and by transesophageal echocardiography, if 
available, at the end of the procedure. The IABP can be exchanged by placing the glide wire through the balloon; removing the balloon over the wire, being sure not to lose the wire position; and then passing a new IABP over the wire while stabilizing the 1-way valve between one's fingers. If this is difficult, it can be done under fluoroscopic guidance. If necessary, the subclavicular incision might need to be reopened in a sterile setting to achieve better control of the 1-way valve and balloon.

This SCA approach has some disadvantages. First, it is best performed in the cardiac catheterization laboratory or the operating room with radiologic screening and with a highquality image intensifier is available. Second, this approach is more time-consuming than the femoral approach and, therefore, is not recommended in an emergency setting in which rapid initiation of support is needed. Finally, although none were observed in this series, a number of potential serious complications can occur, including the risk of stroke-in particular, because the balloon catheter traverses the arch of the aorta—brachial plexus injury, and limb ischemia.

\section{Implications}

The patients described in the present cohort were largely United Network for Organ Sharing status 1A patients. They had significant morbidity; their expected survival in the absence of life-saving intervention could be measured in weeks or even days; and we expected some undesirable outcomes, regardless of management. Nevertheless, from our experience, the SCA balloon pump offers an excellent short-term solution in ambulatory patients with end-stage heart failure, because it preserves mobility while awaiting transplantation or recovery of myocardial function. We did not observe any adverse events related to the placement or use of the SCA-IABP, including stroke secondary to traversing the arch, limb ischemia, bleeding events, thromboembolic events, brachial plexus injuries, or device-related infections. In some instances, the SCA-IABP was an adjunctive measure to support the hemodynamics while a consensus could be reached and a decision made about the suitability for a long-term solution. With increasing experience, we will better define the appropriate criteria for application of this therapy. In particular, although in our series, most patients required a bridge to transplantation, SCAIABPs might have a prophylactic role in the perioperative period for high-risk cardiac surgery patients as a bridge to recovery, particularly in patients with recent myocardial infarction, patients with ventricular septal defects, or patients with a low ejection fraction and mitral valve regurgitation.

\section{Study Limitations}

The limitations of our study included all the caveats of a small, nonrandomized, retrospective case series with limited follow-up. Nevertheless, despite the lack of a comparable technology (in terms of limited invasiveness and cost) for ambulatory patients and given the generally positive outcomes and low likelihood of a randomized study of this approach, these data offer strong validation of this method. However, additional follow-up is needed, and future studies should include prospectively collected data with standardized definitions for neurologic complications and other adverse events and the inclusion of economic outcome measures such as patient satisfaction and cost.

Hemodynamic information was not compiled. In our experience, analysis of hemodynamic data, particularly retrospective data, is often of limited value. These variables are continuously changing over time and there is no standard point when they are available for analysis. Furthermore, with multiple classes of drugs, including vasopressors, inotropes, and anesthetics, as well as multiple drugs within each class, it is difficult to determine the effect of each relative to the device. Finally, analyzing multiple values over time is a statistical challenge. Therefore, the present study did not include hemodynamic data. The hemodynamic benefits of IABP are well established, and no reason exists to expect that this route of insertion would significantly alter the effect of IABPs.

\section{CONCLUSIONS}

The SCA balloon pump can be placed safely, with minimum morbidity in a high risk group of patients, often under local anesthesia. It preserves ambulation when a patient is ambulating preoperatively and allows an out of bed to chair position in more debilitated patients, with obvious benefits to pulmonary function. It is a reasonable option in deteriorating patients in heart failure who are on the transplant list in the short term, up to several weeks. Additional studies are needed to evaluate long-term outcomes, the necessity for anticoagulation, and the cost/benefit ratio of this device compared with alternatives, including LVADs.

\section{References}

1. Kantrowitz A, Tjonneland S, Freed PS, Phillips SJ, Butner AN, Sherman JL Jr. Initial clinical experience with intraaortic balloon pumping in cardiogenic shock. JAMA. 1968;203:135-40.

2. Maccioli GA, Lucas WJ, Norfleet EA. The intra-aortic balloon pump: a review. $J$ Cardiothorac Anesth. 1988;2:365-73.

3. Mayer JH. Subclavian artery approach for insertion of intraaortic balloon. $J$ Thorac Cardiovasc Surg. 1978;76:61-3.

4. McBride LR, Miller LW, Naunheim KS, Pennington DG. Axillary artery insertion of an intraaortic balloon pump. Ann Thorac Surg. 1989;48:874-5.

5. Cochran RP, Starkey TD, Panos AL, Kunzelman KS. Ambulatory intra-aortic balloon pump use as bridge to heart transplant. Ann Thorac Surg. 2002;74: 746-52.

6. Raman J, Loor G, London M, Jolly N. Subclavian artery access for ambulatory balloon pump insertion. Ann Thorac Surg. 2010;90:1032-4

7. Goetz AM, Wagener MM, Miller JM, Muder RR. Risk of infection due to central venous catheters: effect of site of placement and catheter type. Infect Control Hosp Epidemiol. 1998;19:842-5. 